\title{
Классическое магнетосопротивление двухкомпонентной системы, обусловленное термоэлектрическими эффектами
}

\author{
(С) П.С. Алексеев ${ }^{1}$, И.В. Горный ${ }^{1,2,3}$, А.П. Дмитриев ${ }^{1}$, В.Ю. Качоровский ${ }^{1}$, М.А. Семина ${ }^{1}$ \\ ${ }^{1}$ Физико-технический институт им. А.Ф. Иоффе Российской академии наук, \\ 194021 Санкт-Петербург, Россия \\ ${ }^{2}$ Institut für Nanotechnologie, Karlsruhe Institute of Technology, \\ 76021 Karlsruhe, Germany \\ ${ }^{3}$ Institut für Theorie der Kondensierten Materie, Karlsruhe Institute of Technology, \\ 76128 Karlsruhe, Germany \\ E-mail: alekseev_p_s@mail.ru
}

(Получена 5 декабря 2016 г. Принята к печати 18 декабря 2016 г.)

\begin{abstract}
Изучен магнетотранспорт в двумерной двухкомпонентной системе, состоящей из электронов и дырок с одинаковыми концентрациями. Из классического кинетического уравнения выведены уравнения баланса, описывающие перенос носителей и тепла. С помощью решения уравнений баланса для образца в форме длинной полосы рассчитаны распределения концентраций и температур носителей, а также плотности электрического тока. В достаточно большом магнитном поле вблизи краев образца формируются области повышенных и пониженных концентраций, температур и потоков носителей, что приводит к нетривиальному положительному магнетосопротивлению.
\end{abstract}

DOI: 10.21883/FTP.2017.06.44560.8474

\section{1. Введение}

В последние 10 лет были обнаружены новые неожиданные явления в магнетотранспорте двумерных (2D) электронов. В работах [1-4] в полупроводниковых квантовых ямах самого высокого качества наблюдалось гигантское отрицательное магнетосопротивление. Приложение радиочастотного поля к таким образцам приводило к осцилляционной зависимости фотосопротивления от магнитного поля в неквантующих магнитных полях (см., например, обзор [5]). В графене и топологических изоляторах было обнаружено линейное магнетосопротивление в широком интервале классически больших магнитных полей, возникающее при равенстве концентраций электронов и дырок [6-14].

В теории транспортных явлений в полупроводниковых структурах часто предполагается, что неоднородность распределения потоков частиц по образцу несущественна и связь между экспериментально наблюдаемыми величинами токов и напряжений описывается объемным тензором проводимости. Для его расчета используется квантовое или классическое кинетическое уравнение, не содержащее зависимости функции распределения частиц от пространственных координат [5,15-17]. Например, в работах [18-21] были изучены механизмы отрицательного магнетосопротивления и осциллирующей фотопроводимости 2D электронов, обусловленные немарковскими корреляциями (эффектами памяти) при классическом рассеянии электронов на дефектах большого радиуса.

Однако при высоком качестве образца, когда длины релаксации сравнимы с размером образца, приложение внешнего электрического поля может приводить к формированию неоднородных распределений концентраций, температур и потоков частиц. В этом случае ток через образец определяется не только непосредственным дей- ствием электрического и магнитного полей на движение частиц, но и формированием диффузионных потоков частиц, пропорциональных градиентам концентраций, температур и(или) потоков носителей.

В работах [22,23] был изучен магнетотранспорт в двухкомпонентной системе при одинаковых концентрациях электронов и дырок (так называемая точка электронейтральности). Было показано, что в длинном образце за счет процессов рекомбинации и диффузии в магнитном и электрическом полях кроме продольных потоков частиц могут возникать также поперечные потоки. В достаточно сильном магнитном поле вблизи краев образца формируются области, в которых потоки направлены преимущественно вдоль краев. Это приводит к линейной зависимости сопротивления образца от магнитного поля. Анализ экспериментальных данных показывает, что предложенный в [22,23] механизм качественно объясняет наблюдавшееся в работе [14] (и, видимо, в работах [6-13]) линейное магнетосопротивление.

B работах $[24,25]$ изучался электрический транспорт 2D электронов в условиях, когда интенсивность релаксации импульса носителей различна в разных областях образца и неоднородное течение формируется за счет эффекта вязкости. Такой механизм ответствен за гигантское отрицательное магнетосопротивление, наблюдавшееся на квантовых ямах высокого качества в работах [1-4].

В работах [26-29] было показано, что при протекании постоянного тока через образец с металлическими контактами термоэлектрический эффект приводит к возникновению возмущения температуры носителей, которое может быть неоднородным по образцу и вызывает поправку к наблюдаемой величине тока. В работах [34] изучался электрический и тепловой транспорт в однослойном графене в рамках гидродинамической модели, 
то есть в условиях, когда самыми быстрыми являются межчастичные столкновения.

В настоящей статье мы обобщили построенную в [22] теорию магнетотранспорта двухкомпонентной неупорядоченной системы (например, однослойного или многослойного графена) на случай, когда, наряду с процессами рекомбинации и диффузии, существенную роль может играть перенос тепла. Исходя их классического кинетического уравнения, мы вывели балансовые уравнения, описывающие перенос частиц и тепловой энергии. Рассматривается симметричная двухкомпонентная система с одинаковыми свойствами электронов и дырок в режиме электронейтральности (например, однослойный или двухслойный графен). Мы решаем уравнения баланса для образца в форме длинной полосы в режиме, когда скорость рекомбинации много меньше скорости теплообмена. Рассчитаны распределения концентраций, температур и потоков носителей по ширине образца, а также результирующее магнетосопротивление. В достаточно широких образцах возмущение температуры почти не влияет на распределение потоков и концентраций частиц. Магнетосопротивление для таких образцов обусловлено рекомбинацией и диффузией и оказывается линейным в соответствии с рассмотрением работы [22]. Решение балансовых уравнений для достаточно узких образцов приводит к магнетосопротивлению нового типа, связанному с формированием неоднородного распределения температуры носителей. Градиент температуры вызывает дополнительные потоки частиц за счет термоэлектрического эффекта, что приводит к нетривиальному положительному магнетосопротивлению.

\section{2. Кинетическое уравнение}

Рассмотрим двумерную проводящую систему, состоящую из носителей заряда двух типов: электронов и дырок. Особый интерес такая система представляет в случае электронейтральности, когда в равновесии концентрации электронов и дырок одинаковы. Мы будем считать, что энергетический спектр и релаксационные характеристики электронов и дырок также одинаковы.

Введем следующие обозначения. Заряды электронов и дырок $q^{\alpha}, \alpha=e, h$, будем записывать через абсолютную величину элементарного заряда $e>0: q^{e}=-e$, $q^{h}=e$. Энергетический спектр носителей, $\varepsilon_{p}^{e}=\varepsilon_{p}^{h}=\varepsilon_{p}$, считаем изотропным относительно направлений импульса носителей $\mathbf{p}=p(\cos \varphi, \sin \varphi)$. Абсолютную величину импульса $p$ можно записать как функцию энергии: $p(\varepsilon), \varepsilon_{p(\varepsilon)}=\varepsilon$. Скорость носителей $\mathbf{v}=\partial \varepsilon_{p} / \partial \mathbf{p}$ сонаправлена с импульсом р и имеет абсолютную величину $v(p)=\partial \varepsilon_{p} / \partial p, v(\varepsilon)=v(p(\varepsilon))$. Равновесные концентрации электронов и дырок имеют вид

$$
n_{0}=\int d \Gamma_{\mathbf{p}} f_{\mathrm{F}}\left(\varepsilon_{p}\right)=\int d \varepsilon v(\varepsilon) f_{\mathrm{F}}(\varepsilon),
$$

где $d \boldsymbol{\Gamma}_{\mathbf{p}}=N_{g} d^{2} \mathbf{p} /(2 \pi \hbar)^{2}, N_{g}$ - фактор вырождения, равный 4 для однослойного и двухслойного графена, $v(\varepsilon)-$ плотность состояний электронов и дырок, фермиевская функция распределения $f_{\mathrm{F}}(\varepsilon)=1 /\left[1+e^{\left(\varepsilon-\mu_{0}\right) / T_{0}}\right]$ сооответствует равновесным значениям химического потенциала $\mu_{0}$ и температуры $T_{0}$, одинаковым для электронов и дырок.

Неравновесные функции распределения электронов и дырок $f^{\alpha}(\mathbf{p})$ в каждой точке образца могут быть представлены в виде суммы изотропной и анизотропной частей (относительно направлений р):

$$
f^{\alpha}(\mathbf{p}, \mathbf{r})=f_{\text {is }}^{\alpha}(\varepsilon, \mathbf{r})+f_{\text {an }}^{\alpha}(\varepsilon, \varphi, \mathbf{r}) .
$$

Неравновесные поправки к концентрациям и температурам носителей связаны с изотропными частями функций распределения $f_{\text {is }}^{\alpha}$; анизотропные части $f_{\text {an }}^{\alpha}$ описывают неравновесные потоки частиц, вызванные внешними полями, электрическим $\mathbf{E}$ и магнитным $\mathbf{B}$.

Мы будем изучать возмущения системы, которые не нарушают симметрию относительно замены электронов на дырки и изменения знака всех зарядов. Для таких возмущений симметричные части функций рапределения электронов и дырок одинаковы: $f_{\text {is }}^{e}=f_{\text {is }}^{h}=f_{\text {is. }}$ Анизотропные части функций распределения электронов и дырок различны: $f_{\text {an }}^{e} \neq f_{\text {an }}^{h}$. Различие $f_{\text {an }}^{e}$ и $f_{\text {an }}^{h}$ обусловлено тем, что электрическое поле действует на электроны и дырки в противоположных направлениях. Сосуществование зарядовой нейтральности в каждой точке $\mathbf{r}$ и ненулевого электрического поля, стремящегося разделить в пространстве электроны и дырки, т.е. нарушить равенство $f_{\text {is }}^{e}(\varepsilon, \mathbf{r})=f_{\text {is }}^{h}(\varepsilon, \mathbf{r})$, возможно при следующем условии. Электрическое поле должно быть перпендикулярно градиенту от изотропных частей функций распределения $f_{\text {is }}(\varepsilon, \mathbf{r})$ при любых значениях $\varepsilon$ и $\mathbf{r}$

$$
\mathbf{E}(\mathbf{r}) \cdot \frac{\partial f_{\text {is }}(\varepsilon, \mathbf{r})}{\partial \mathbf{r}}=0 .
$$

Мы исследуем линейный отклик, возникающий на внешнее электрическое поле $\mathbf{E}$. В такой ситуации основная часть функций распределения электронов и дырок $f^{\alpha}$ есть фермиевская функция $f_{\mathrm{F}}$, поправка к ней $\delta f^{\alpha}$ пропорциональна приложенному полю: $f^{\alpha}=f_{\mathrm{F}}+\delta f^{\alpha}, \delta f^{\alpha} \propto E$. Для введенных в (2) симметричных и асимметричных частей функций распределения мы получаем: $f_{\text {is }}=f_{\mathrm{F}}+\delta f_{\text {is }}, f_{\text {an }}^{\alpha} \equiv \delta f_{\text {an }}^{\alpha}$, $\delta f^{\alpha}=\delta f_{\text {is }}+\delta f_{\text {an }}^{\alpha}$.

Рассмотрим систему в режиме, когда характерные времена релаксации удовлетворяют условию

$$
\tau \ll \tau_{e e} \ll \tau_{\mathrm{ph}},
$$

где $\tau_{\mathrm{ph}}-$ время термализации носителей за счет взаимодействия носителей с фононами, $\tau_{e e}-$ время термализации носителей за счет межчастичного взаимодействия, $\tau-$ время релаксации $f_{\mathrm{an}}^{\alpha}$, определяемое преимущественно рассеянием носителей на беспорядке.

Условие (4) приводит к тому, что изотропные части функций распределения электронов и дырок $f_{\text {is }}$ являют- 
ся локально-равновесной фермиевской функцией

$$
f_{\text {is }}(\varepsilon, \mathbf{r})=\frac{1}{1+e^{\frac{\varepsilon-\mu(\mathbf{r})}{T(\mathbf{r})}}}
$$

формирующейся за счет быстрых межчастичных столкновений. Здесь температура $T(\mathbf{r})$ и химический потенциал $\mu(\mathbf{r})$ отличны от невозмущенных значений $T_{0}$ и $\mu_{0}$. Более медленные процессы взаимодействия с фононами приводят к релаксации температуры носителей $T$ к температуре решетки $T_{0}$. Пусть возмущения химического потенциала, $\delta \mu(\mathbf{r})=\mu(\mathbf{r})-\mu_{0}$, и температуры, $\delta T(\mathbf{r})=T(\mathbf{r})-T_{0}$, малы по сравнению с величиной $\max \left(\mu_{0}, T_{0}\right)$. Тогда изотропные части функций распределения в линейном приближении по возмущениям имеют вид $f_{\text {is }}=f_{\mathrm{F}}+\delta f_{\text {is, }}$ где

$$
\delta f_{\text {is }}(\varepsilon, \mathbf{r})=-f_{\mathrm{F}}^{\prime}(\varepsilon) \delta \mu(\mathbf{r})-f_{\mathrm{F}}^{\prime}(\varepsilon) \frac{\varepsilon-\mu_{0}}{T_{0}} \delta T(\mathbf{r}) .
$$

Мы будем изучать эффекты, связанные только с первыми угловыми гармониками анизотропных частей функций распределения, т.е. мы везде будем предполагать, что $\delta f_{\text {an }}^{\alpha}(\varepsilon, \varphi)=C^{\alpha}(\varepsilon) \cos (\varphi)+S^{\alpha}(\varepsilon) \sin (\varphi)$. Условие (4) означает, что релаксация $\delta f_{\text {an }}^{\alpha}$ - самая быстрая. Поэтому значения $\delta f_{\text {an }}^{\alpha}$ малы в меру величины $\tau$ : $\delta f_{\text {an }}^{\alpha} \propto \tau$, что приводит к соотношению $\delta f_{\text {an }}^{\alpha} \ll \delta f_{\text {is. }}$.

Уравнения Больцмана для функций $f^{e}$ и $f^{h}$ имеют вид

$$
\begin{aligned}
\frac{\partial f^{\alpha}}{\partial t} & +\mathbf{v} \frac{\partial f^{\alpha}}{\partial \mathbf{r}}+\mathbf{F}^{\alpha} \frac{\partial f^{\alpha}}{\partial \mathbf{p}} \\
& =\mathrm{St}_{e e}^{\alpha}\left[f^{\alpha}, f^{\bar{\alpha}}\right]+\operatorname{St}_{\mathrm{ph}}^{\alpha}\left[f^{\alpha}, f^{\bar{\alpha}}\right]+\operatorname{St}_{\text {imp }}^{\alpha}\left[f^{\alpha}\right],
\end{aligned}
$$

где символ $\bar{\alpha}$ означает $h$ при $\alpha=e$ и $e$ при $\alpha=h$. Силы $\mathbf{F}^{\alpha}(\mathbf{r})$ при наличии электрического поля $\mathbf{E}(\mathbf{r})$ в плоскости $x y$ и магнитного поля $\mathbf{B}=B \mathbf{e}_{z}$ имеют вид $\mathbf{F}^{\alpha}=q^{\alpha}\left(E_{x}-B v_{y} / c, E_{y}+B v_{x} / c\right)$. Интегралы столкновений $\mathrm{St}_{e e}^{\alpha}, \mathrm{St}_{\mathrm{ph}}^{\alpha}$ и $\mathrm{St}_{\mathrm{imp}}^{\alpha}$ описывают взаимодействие носителей друг с другом, с фононами и с беспорядком. Считаем все интегралы столкновений операторами, линеаризованными по $\delta f^{\alpha}$ вблизи $f_{0}^{\alpha}=f_{\mathrm{F}}$.

Рассеяние на беспорядке является упругим, поэтому интегралы столкновений $\mathrm{St}_{\mathrm{imp}}^{\alpha}$ зависят только от функции распределения носителей данного типа $f^{\alpha}$. При этом изотропные части функций распределения не релаксируют за счет взаимодействия с беспорядком, $\mathrm{St}_{\text {imp }}^{\alpha}\left[f_{\text {is }}\right]=0$, а анизотроные части $\delta f_{\text {an }}^{\alpha}(\varepsilon, \varphi)$ при различных энергиях $\varepsilon$ релаксируют независимо. В случае, когда $\delta f_{\text {an }}^{\alpha}$ содержит только первую гармонику, оператор $\mathrm{St}_{\mathrm{imp}}^{\alpha}$ принимает хорошо известный диагональный по $\varepsilon$ и $\varphi$ вид

$$
\mathrm{St}_{\mathrm{imp}}^{\alpha}\left[\delta f_{\mathrm{an}}^{\alpha}\right]=-\frac{\delta f_{\mathrm{an}}^{\alpha}(\varepsilon, \varphi)}{\tau(\varepsilon)} .
$$

Межчастичные и электрон-фононные интегралы столкновений $\mathrm{St}_{e e, \mathrm{ph}}^{\alpha}$, во-первых, состоят из слагаемых, описывающих процессы без рождения и гибели частиц (слагаемые типа „(1)“). Такие слагаемые для оператора
$\mathrm{St}_{e e}^{\alpha}$ учитывают столкновения двух электронов или двух дырок, а для оператора $\mathrm{St}_{\mathrm{ph}}^{\alpha}-$ рассеяние электрона или дырки на фононах. Слагаемые типа „(1)“ действуют только на функцию распределения данного типа частиц $\alpha$ и сохраняют полное число частиц. Во-вторых, $\mathrm{St}_{e e}^{\alpha}$ и $\mathrm{St}_{\mathrm{ph}}^{\alpha}$ содержат вклады от процессов, сопровождающихся рождением и гибелью электронно-дырочных пар (слагаемые типа „(2)“). Для оператора $\mathrm{St}_{e e}^{\alpha}$ это процессы оже-рекомбинации с участием электроннодырочной пары и дополнительного электрона или дырки; для оператора $\mathrm{St}_{\mathrm{ph}}^{\alpha}$ это процессы фононной рекомбинации с участием электронно-дырочной пары и фонона. Слагаемые типа „, $(2)^{\text {“ }}$ в $\mathrm{St}_{e e}^{\alpha} \quad$ и $\mathrm{St}_{\mathrm{ph}}^{\alpha}$ зависят как от функции распределения частиц данного типа $\alpha$, так и от функции распределения частиц другого типа $\bar{\alpha}$, и не сохраняют концентрации электронов и дырок. Таким образом, имеем:

$$
\begin{gathered}
\mathrm{St}_{e e, \mathrm{ph}}^{\alpha}\left[f^{\alpha}, f^{\bar{\alpha}}\right]=\mathrm{St}_{e e, \mathrm{ph}}^{(1)}\left[f^{\alpha}\right]+\mathrm{St}_{e e, \mathrm{ph}}^{\alpha,(2)}\left[f^{\alpha}, f^{\bar{\alpha}}\right], \\
\int d \Gamma_{\mathbf{p}} \mathrm{St}_{e e, \mathrm{ph}}^{(1)}\left[f^{\alpha}\right](\mathbf{p})=0 .
\end{gathered}
$$

Изотропные равновесные части функций распределения $f_{\text {is }}$ вида (5) не релаксируют за счет процессов межчастичных столкновений: $\mathrm{St}_{e e}^{(1)}\left[f_{\text {is }}\right]=0$. Заметим, что анизотропные функции распределения вида $f_{\text {an,eq }}^{\alpha}(\mathbf{p}, \mathbf{r})=f_{\mathrm{F}}^{\prime}\left(\varepsilon_{p}\right) \mathbf{p} \cdot \mathbf{V}^{\alpha}(\mathbf{r})$ также не релаксируют за счет межчастичных столкновений: $\mathrm{St}_{e e}^{(1)}\left[f_{\text {an,eq }}^{\alpha}\right]=0$. Выражения $f_{\text {an,eq }}^{\alpha}$ есть анизотропные части функций распределения, которые являются равновесными в системах отсчета, движущихся со скоростями $\mathbf{V}^{\alpha}$; они отвечают наличию потоков частиц, но отсутствию потоков тепла. При межчастичных столкновениях сохраняются также импульс и энергия пары сталкивающихся частиц, поэтому для скоростей релаксации суммарных импульса и энергии за счет межчастичных столкновений имеем

$$
\int d \Gamma_{\mathbf{p}} \mathbf{p S t}_{e e}^{(1)}\left[f^{\alpha}\right](\mathbf{p})=0, \quad \int d \Gamma_{\mathbf{p}} \varepsilon_{p} \mathrm{St}_{e e}^{(1)}\left[f^{\alpha}\right](\mathbf{p})=0 .
$$

При взаимодействии электронов и дырок с фононами в процессах типа „(1)“ сохраняется число частиц (см. (10)), но происходит релаксация энергии. Поэтому для локально-равновесных изотропных функций распределения вида (6) мы получаем:

$$
\begin{gathered}
\int d \Gamma_{\mathbf{p}} \varepsilon_{p} \mathrm{St}_{\mathrm{ph}}^{(1)}\left[\delta f_{\text {is }}\right](\mathbf{p})=-L \delta T, \\
\mathrm{St}_{\mathrm{ph}}^{(1)}\left[-f_{\mathrm{F}}^{\prime}\left(\varepsilon_{p^{\prime}}\right) \delta \mu\right]=0,
\end{gathered}
$$

где константа $L=L\left(T_{0}\right)$ характеризует скорость теплообмена с фононами и имеет вид

$$
L=\int d \Gamma_{\mathbf{p}} \varepsilon_{p} \mathrm{St}_{\mathrm{ph}}^{(1)}\left[f_{\mathrm{F}}^{\prime}\left(\varepsilon_{p^{\prime}}\right) \frac{\varepsilon_{p^{\prime}}-\mu_{0}}{T_{0}}\right](\mathbf{p}) .
$$

К соотношениям (10), (11) и (12) приводит, например, интеграл столкновений, описывающий взаимодействие 
двумерных электронов (или дырок) с трехмерными акустическими фононами:

$$
\begin{aligned}
\mathrm{St}_{\mathrm{ph}}^{(1)} & \delta f](\mathbf{p})=\int d \Gamma_{\mathbf{p}^{\prime}} M_{\mathbf{p p}^{\prime}} \\
& \times\left[-F_{-}\left(\varepsilon_{p}, \varepsilon_{p^{\prime}}\right) \delta f(\mathbf{p})+F_{+}\left(\varepsilon_{p}, \varepsilon_{p^{\prime}}\right) \delta f\left(\mathbf{p}^{\prime}\right)\right],
\end{aligned}
$$

где $M_{\mathbf{p p}^{\prime}}=M\left(\varepsilon_{p}, \mathbf{p}-\mathbf{p}^{\prime}\right)-$ вероятность перехода электрона между состояниями $\mathbf{p}$ и $\mathbf{p}^{\prime}$ с излучением или поглощением фонона, усредненная по волновому вектору фонона в направлении $z$,

$$
\begin{gathered}
F_{-}\left(\varepsilon, \varepsilon^{\prime}\right)=N_{B}\left(\left|\varepsilon-\varepsilon^{\prime}\right|\right)+\mid \begin{array}{l}
1-f_{\mathrm{F}}\left(\varepsilon^{\prime}\right), \quad \varepsilon^{\prime}<\varepsilon \\
f_{\mathrm{F}}\left(\varepsilon^{\prime}\right), \quad \varepsilon^{\prime}>\varepsilon
\end{array} \\
F_{+}\left(\varepsilon, \varepsilon^{\prime}\right)=N_{B}\left(\left|\varepsilon-\varepsilon^{\prime}\right|\right)+\mid \begin{array}{l}
f_{\mathrm{F}}(\varepsilon), \quad \varepsilon^{\prime}<\varepsilon \\
1-f_{\mathrm{F}}(\varepsilon), \quad \varepsilon^{\prime}>\varepsilon
\end{array}
\end{gathered}
$$

$N_{B}(E)=1 /\left[e^{E / T_{0}}-1\right]-$ функция распределения равновесных фононов с температурой $T_{0}$. Свойства ядра $M(\varepsilon, \Delta \mathbf{p})$ зависят от соотношения между температурой и различными характеристиками 2D электронов (см. работы [30-32]).

Релаксация анизотропных частей функций распределения $\delta f_{\text {an }}^{\alpha}$, содержащих только первую угловую гармонику, за счет межчастичных и электрон-фононных процессов типа „(1)“6 описывается оператором вида

$$
\sum_{\sigma=e e, \mathrm{ph}} \mathrm{St}_{\sigma}^{(1)}\left[\delta f_{\mathrm{an}}^{\alpha}\right](\varepsilon, \varphi)=\int d \varepsilon^{\prime} W_{\varepsilon \varepsilon^{\prime}}^{(1)} \delta f_{a}^{\alpha}\left(\varepsilon^{\prime}, \varphi\right) .
$$

Однако в силу условия (4) этим вкладом в релаксацию $\delta f_{\text {an }}^{\alpha}$ можно пренебречь по сравнению с примесным вкладом (8).

Слагаемые типа „(2)“ межчастичного и электронфононного интегралов столкновений, описывающие процессы с рождением и гибелью частиц, представляют собой операторы вида

$$
\begin{aligned}
& \sum_{\sigma=e e, \mathrm{ph}} \operatorname{St}_{\sigma}^{\alpha,(2)}\left[\delta f^{\alpha}, \delta f^{\bar{\alpha}}\right](\mathbf{p}) \\
& \quad=\int d \Gamma_{\mathbf{p}^{\prime}}\left[W_{\mathbf{p p}}^{s} \delta f^{\alpha}\left(\mathbf{p}^{\prime}\right)+W_{\mathbf{p p}^{\prime}}^{o} \delta f^{\bar{\alpha}}\left(\mathbf{p}^{\prime}\right)\right],
\end{aligned}
$$

где ядра $W_{\mathbf{p p}^{\prime}}^{s}$ и $W_{\mathbf{p p}^{\prime}}^{o}$, аналогично (13), содержат слагаемые ухода и прихода частиц. Оператор (15) приводит к релаксации как изотропных, так и анизотропных частей функций распределения $\delta f^{\alpha}=\delta f_{\text {is }}+\delta f_{\text {an }}^{\alpha}$. Однако в силу соотношения (4) релаксация $\delta f_{\text {an }}^{\alpha}$ определяется преимущественно рассеянием на беспорядке. Поэтому в операторе (15) будем учитывать только те слагаемые, которые содержат $\delta f_{\text {is }}$ и приводят к изменению числа частиц в изучаемом состоянии $(\alpha, \mathbf{p})$. Например, для таких слагаемых в операторе $\mathrm{St}_{\mathrm{ph}}^{\alpha,(2)}$ можно вывести выражение

$$
\begin{aligned}
& \mathrm{St}_{\mathrm{ph}}^{\alpha,(2)}\left[\delta f_{\text {is }}, \delta f_{\text {is }}\right](\mathbf{p})=- \int d \Gamma_{\mathbf{p}^{\prime}} W_{\mathbf{p p}^{\prime}}^{(2)} \\
& \times\left[G_{p p^{\prime}} \delta f_{\text {is }}\left(\varepsilon_{p}\right)+H_{p p^{\prime}} \delta f_{\text {is }}\left(\varepsilon_{p^{\prime}}\right)\right], \\
& G_{p p^{\prime}}=N_{B}\left(\left|\Delta \varepsilon_{p p^{\prime}}\right|\right)+\mid \begin{array}{l}
f_{F}\left(\varepsilon_{p^{\prime}}\right), \Delta \varepsilon_{p p^{\prime}}>0 \\
1-f_{F}\left(\varepsilon_{p^{\prime}}\right), \Delta \varepsilon_{p p^{\prime}}<0
\end{array} \\
& H_{p p^{\prime}}=N_{B}\left(\left|\Delta \varepsilon_{p p^{\prime}}\right|\right)+\mid \begin{array}{l}
f_{F}\left(\varepsilon_{p}\right), \Delta \varepsilon_{p p^{\prime}}>0 \\
1-f_{F}\left(\varepsilon_{p}\right), \Delta \varepsilon_{p p^{\prime}}<0
\end{array}
\end{aligned}
$$

где $\Delta \varepsilon_{p p^{\prime}}=\varepsilon_{p}-\mu_{0}+\varepsilon_{p^{\prime}}-\mu_{0}-$ энергия фонона, $W_{\mathbf{p p}^{\prime}}^{(2)}=W_{\mathbf{p}^{\prime} \mathbf{p}}^{(2)}-$ вероятность рекомбинации электроннодырочной пары с импульсами р и $\mathbf{p}^{\prime}$ за счет электронфононного взаимодействия.

В дальнейшем нам потребуются скорости релаксации концентраций и тепловых энергий частиц за счет рассеяния. Эти величины вычисляются путем суммирования по р интегралов столкновений $\mathrm{St}_{\sigma}^{\alpha}, \sigma=\mathrm{ph}, e e$, умноженных на единицу или на величину $\varepsilon_{p}-\mu_{0}$. С учетом выражения (16) скорости релаксации концентраций и тепловых энергий за счет электрон-фононных процессов типа ,(2)“ при $\delta f_{\text {is }}$ вида (6) даются формулами

$$
\begin{gathered}
\int d \Gamma_{\mathbf{p}} \mathrm{St}_{\mathrm{ph}}^{\alpha,(2)}\left[\delta f_{\text {is }}, \delta f_{\text {is }}\right]\left(\varepsilon_{p}\right)=-R \delta \mu-R^{\varepsilon} \delta T, \\
\int d \Gamma_{\mathbf{p}}\left(\varepsilon_{p}-\mu_{0}\right) \operatorname{St}_{\mathrm{ph}}^{\alpha,(2)}\left[\delta f_{\text {is }}, \delta f_{\text {is }}\right]\left(\varepsilon_{p}\right)=-T_{0} R^{\varepsilon} \delta \mu-R^{\varepsilon \varepsilon} \delta T,
\end{gathered}
$$

где коэффициенты разложения можно привести к виду

$$
\begin{gathered}
\left(\begin{array}{c}
R \\
R^{\varepsilon} \\
R^{\varepsilon \varepsilon}
\end{array}\right)=\iint d \Gamma_{\mathbf{p}} d \Gamma_{\mathbf{p}^{\prime}} K_{\mathbf{p p}^{\prime}}\left(\begin{array}{c}
2 \\
\Delta \varepsilon_{p p^{\prime}} / T_{0} \\
\Delta \varepsilon_{p p^{\prime}}^{2} / 2 T_{0}
\end{array}\right), \\
K_{\mathbf{p p}^{\prime}}=K_{\mathbf{p}^{\prime} \mathbf{p}}=W_{\mathbf{p p}^{\prime}}^{(2)}\left[1-f_{\mathrm{F}}\left(\varepsilon_{p}\right)\right]\left[1-f_{\mathrm{F}}\left(\varepsilon_{p^{\prime}}\right)\right] N_{B}\left(\Delta \varepsilon_{p p^{\prime}}\right) .
\end{gathered}
$$

Эти формулы соответствуют наличию энергетической щели между состояниями электронов и дырок, когда для всех $p$ и $p^{\prime}$ выполнено неравенство $\Delta \varepsilon_{p p^{\prime}}>0$. Формулы для случая бесщелевой двухкомпонентной системы, когда возможны оба неравенства $\Delta \varepsilon_{p p^{\prime}}>0$ и $\Delta \varepsilon_{p p^{\prime}}<0$, имееют более громоздкий вид, поэтому мы их не приводим. Величины $R, R^{\varepsilon}, R^{\varepsilon \varepsilon}$ характеризуют скорости рекомбинации. Примечательно, что первое и второе уравнения (17) содержат один и тот же коэффициент $R^{\varepsilon}$; это есть микроскопическое выражение принципа Онсагера симметрии кинетических коэффициентов.

Соотношение, аналогичное (17), будет справедливо и для усредненных скоростей релаксации за счет действия межчастичного оператора $\mathrm{St}_{e e}^{\alpha,(2)}$. Поэтому далее мы считаем, что $R, R^{\varepsilon}, R^{\varepsilon \varepsilon}$ учитывают рекомбинацию за счет как электрон-фононного, так и межчастичного взаимодействий. 
Уравнения Больцмана (7) для $\delta f^{\alpha}=\delta f_{\text {an }}^{\alpha}+\delta f_{\text {is }}$ содержат члены двух типов: изотропные и анизотропные относительно направлений р. Пусть время релаксации $\tau(\varepsilon)$ в примесном интеграле столкновений (8) много меньше всех остальных времен релакасации, а также характерного времени изменения внешнего поля. Тогда с помощью уравнения (7) можно показать, что выполнено неравенство $\delta f_{\text {an }}^{\alpha} \ll \delta f_{\text {is. }}$ Благодаря этому неравенству в анизотропных слагаемых уравнения (7) функции $\delta f_{\text {an }}^{\alpha}$ следует удержать только в слагаемых: $-\delta f^{\alpha} / \tau(\varepsilon)$ и $\left(q^{\alpha} B / c\right)\left[\mathbf{v} \times \mathbf{e}_{z}\right] \cdot\left[\partial f^{\alpha} / \partial \mathbf{p}\right]$. Последнее слагаемое можно переписать в виде $-\omega_{c}^{\alpha}(\varepsilon)\left[\partial f^{\alpha} / \partial \varphi\right]$, где $\omega_{c}^{\alpha}(\varepsilon)=q^{\alpha} B v(\varepsilon) / c p(\varepsilon)$ - циклотронная частота. Таким образом, анизотропная часть (7) представляет собой дифференциальное уравнение по переменной $\varphi$ для поиска $\delta f_{\text {an }}^{\alpha}$. Решение этого уравнения приводит к следующему соотношению между $\delta f_{\text {an }}^{\alpha}$ и $\delta f$ is:

$$
\begin{aligned}
\delta f_{\text {an }}^{\alpha}(\varepsilon, \varphi, \mathbf{r})= & -\frac{\tau}{1+\omega_{c}^{2} \tau^{2}} \mathbf{v}^{\mathrm{T}}\left(\begin{array}{cc}
1 & \omega_{c}^{\alpha} \tau \\
-\omega_{c}^{\alpha} \tau & 1
\end{array}\right) \\
& \times\left[\frac{\partial}{\partial \mathbf{r}}+q^{\alpha} \mathbf{E} \frac{\partial}{\partial \varepsilon}\right] f_{\text {is }}(\varepsilon, \mathbf{r}),
\end{aligned}
$$

где $\quad \tau=\tau(\varepsilon), \quad \omega_{c}^{\alpha}=\omega_{c}^{\alpha}(\varepsilon), \quad \omega_{c}=\left|\omega_{c}^{\alpha}(\varepsilon)\right|, \quad \mathbf{v}=v(\varepsilon)$ $\times[\cos (\varphi), \sin (\varphi)]$.

Для линейных по электрическому полю $\mathbf{E}$ возмущений мы имеем $\delta f_{\text {is }}, \delta f_{\text {an }}^{\alpha} \propto E$. Поле $\mathbf{E}$ явно входит в уравнении (7) только в слагаемое $q^{\alpha} \mathbf{E}\left[\partial f^{\alpha} / \partial \mathbf{p}\right]$, поэтому в этом слагаемом нужно учесть только равновесную невозмущенную часть функции распределения $f_{0}^{\alpha}=f_{\mathrm{F}}$. С учетом этого соображения и выражения (6) для $f_{\text {is }}$ из уравнения (18) окончательно получаем:

$$
\begin{aligned}
\delta f_{\mathrm{an}}^{\alpha}(\varepsilon, \varphi, \mathbf{r}) & =\frac{-f_{\mathrm{F}}^{\prime}(\varepsilon) \tau}{1+\omega_{c}^{2} \tau^{2}} \mathbf{v}^{\mathrm{T}}\left(\begin{array}{cc}
1 & \omega_{c}^{\alpha} \tau \\
-\omega_{c}^{\alpha} \tau & 1
\end{array}\right) \\
\times & {\left[-\nabla \delta \mu(\mathbf{r})-\frac{\varepsilon-\mu_{0}}{T_{0}} \nabla \delta T(\mathbf{r})+q^{\alpha} \mathbf{E}\right] . }
\end{aligned}
$$

\section{3. Вывод уравнений баланса}

Для дальнейшего рассмотрения удобно ввести поток частиц данного вида при фиксированной энергии:

$$
\mathbf{j}^{\alpha}(\varepsilon, \mathbf{r})=\int_{0}^{2 \pi} \frac{d \varphi}{2 \pi} \mathbf{v}(\varepsilon, \varphi) \delta f_{\text {an }}^{\alpha}(\varepsilon, \varphi, \mathbf{r})
$$

На основе этого выражения легко вычислить потоки частиц данного вида $\mathbf{j}^{\alpha}$ и потоки тепловой энергии $\mathbf{q}^{\alpha}$, переносимой частицами данного вида $\alpha$ :

$\mathbf{j}^{\alpha}(\mathbf{r})=\int d \varepsilon v(\varepsilon) \mathbf{j}(\varepsilon, \mathbf{r}), \mathbf{q}^{\alpha}(\mathbf{r})=\int d \varepsilon v(\varepsilon) \mathbf{j}(\varepsilon, \mathbf{r})\left(\varepsilon-\mu_{0}\right)$.
Из выражений (19) и (20) получаем

$$
\mathbf{j}^{\alpha}(\varepsilon, \mathbf{r})=\hat{\sigma}^{\alpha}(\varepsilon)\left[-\nabla \delta \mu(\mathbf{r})-\frac{\varepsilon-\mu_{0}}{T_{0}} \nabla \delta T(\mathbf{r})+q^{\alpha} \mathbf{E}\right],
$$

где введен тензор проводимости при данной энергии:

$$
\hat{\sigma}^{\alpha}(\varepsilon)=\frac{-f_{\mathrm{F}}^{\prime}(\varepsilon) \tau}{1+\omega_{c}^{2} \tau^{2}} \frac{v(\varepsilon)^{2}}{2}\left(\begin{array}{cc}
1 & \omega_{c}^{\alpha} \tau \\
-\omega_{c}^{\alpha} \tau & 1
\end{array}\right) .
$$

Используя (21) и (22), выразим потоки $\mathbf{j}^{\alpha}(\mathbf{r})$ и $\mathbf{q}^{\alpha}(\mathbf{r})$ через электрическое поле, градиенты химического потенциала и температуры:

$$
\begin{aligned}
& \mathbf{j}^{\alpha}=\hat{\sigma}^{\alpha}\left(q^{\alpha} \mathbf{E}-\nabla \delta \mu\right)-\hat{\alpha}^{\alpha} \nabla \delta T, \\
& \mathbf{q}^{\alpha}=\hat{\beta}^{\alpha}\left(q^{\alpha} \mathbf{E}-\nabla \delta \mu\right)-\hat{\kappa}^{\alpha} \nabla \delta T .
\end{aligned}
$$

Здесь введены следующие обозначения для коэффициентов проводимости, термоэлектрических коэффициентов и коэффициентов теплопроводности:

$$
\left(\begin{array}{l}
\hat{\sigma}^{\alpha} \\
\hat{\alpha}^{\alpha} \\
\hat{\beta}^{\alpha} \\
\hat{\kappa}^{\alpha}
\end{array}\right)=\int d \varepsilon v(\varepsilon) \hat{\sigma}^{\alpha}(\varepsilon)\left(\begin{array}{c}
1 \\
\left(\varepsilon-\mu_{0}\right) / T_{0} \\
\varepsilon-\mu_{0} \\
\left(\varepsilon-\mu_{0}\right)^{2} / T_{0}
\end{array}\right) .
$$

Из формул (26) видно, что выполняется соотношение Онсагера для термоэлектрических коэффициентов: $\hat{\beta}^{\alpha}=T_{0} \hat{\alpha}^{\alpha}$.

Для вывода соотношения (18) между $\delta f_{\text {an }}^{\alpha}$ и $\delta f_{\text {is }}$ и следующих из него формул (24) и (25) для потоков $\mathbf{j}^{\alpha}$ и $\mathbf{q}^{\alpha}$ мы использовали только анизотропную часть уравнений Больцмана (7). Теперь используем изотропную часть (7) для получения итоговых уравнений для изотропных частей $\delta f_{\text {is }}$ функций распределения $f^{\alpha}$. Искомые уравнения являются уравнениями баланса концентраций частиц и переносимой ими тепловой энергии. Они и выражения (24) и (25) составят полную систему уравнений для нахождения величин $\delta \mu, \delta T, \mathbf{j}^{\alpha}$ и $\mathbf{q}^{\alpha}$.

Для получения уравнений баланса концентраций усредним (7) по импульсу р с учетом соотношений (17) для $\mathrm{St}_{\mathrm{ph}}^{\alpha,(2)}$ и аналогичных соотношений для $\mathrm{St}_{e e}^{\alpha,(2)} . \mathrm{Xa-}$ рактерное время изменения макроскопических величин и неупругие времена релаксации могут быть сравнимы между собой. Мы получим:

$$
\frac{\partial \delta n}{\partial t}+\operatorname{divj}^{\alpha}=-R \delta \mu-R^{\varepsilon} \delta T
$$

где $\delta n=\int d \varepsilon v(\varepsilon) \delta f_{\text {is }}(\varepsilon)$ - неравновесная часть концентрации электронов и дырок. Эти уравнения имеют прозрачный физический смысл. Они описывают изменения концентраций частиц каждого сорта $\alpha$ за счет наличия потоков $\mathbf{j}^{\alpha}(\mathbf{r})$ и за счет рождения и гибели частиц при рекомбинации. Усредняя кинетическое 
уравнение (7), умноженное на $\varepsilon_{p}-\mu_{0}$, мы получим уравнения баланса тепловой энергии:

$\frac{\partial \delta n_{\varepsilon}}{\partial t}+\operatorname{divq}^{\alpha}-q^{\alpha} \mathbf{E} \cdot \mathbf{j}^{\alpha}=-T_{0} R^{\varepsilon} \delta \mu-R^{\varepsilon \varepsilon} \delta T-L \delta T$,

где $\delta n_{\varepsilon}=\int d \varepsilon v(\varepsilon)\left(\varepsilon-\mu_{0}\right) \delta f_{\text {is }}(\varepsilon) \quad$ - неравновесная плотность тепловой энергии электронов и дырок. Слагаемое $-q^{\alpha} \mathbf{E} \cdot \mathbf{j}^{\alpha}$ описывает работу внешних сил в единицу времени по перемещению зарядов (т.е. рассеиваемое джоулево тепло). При изучении линейного отклика на поле $\mathbf{E}$ это слагаемое не нужно учитывать.

Мы рассматриваем возмущения, сохраняющие электронно-дырочную симметрию системы в каждой точке, т.е. наряду с соотношениями $\delta \mu^{e}=\delta \mu^{h}, \delta T^{e}=\delta T^{h}$, $\delta n^{e}=\delta n^{h}$ должно быть выполнено $\delta n_{\varepsilon}^{e}=\delta n_{\varepsilon}^{h}$. Однако в уравнениях (27) и (28) слагаемые $\operatorname{div} \mathbf{j}^{\alpha}, \operatorname{div} \mathbf{q}^{\alpha}$ и $-e^{\alpha} \mathbf{E} \cdot \mathbf{j}^{\alpha}$ могут, в принципе, зависеть от типа частиц $\alpha=e, h$. Можно показать, что независимость этих слагаемых от $\alpha$ обеспечивается видом выражений для потоков (24) и (25), видом коэффициентов (26) и условием существования зарядово-нейтрального возмущения (3), которое ввиду разложения (6) сводится к соотношениям $\mathbf{E} \cdot \nabla \delta \mu=0$ и $\mathbf{E} \cdot \nabla \delta T=0$.

Возмущения концентраций и плотностей тепловых энергий частиц $\delta n$ и $\delta n_{\varepsilon}$ выражаются как линейные комбинации $\delta \mu$ и $\delta T, \quad \delta n=a \delta \mu+a^{\varepsilon} \delta T$, $\delta n_{\varepsilon}=T_{0} a^{\varepsilon} \delta \mu+a^{\varepsilon \varepsilon} \delta T$, с коэффициентами

$$
\left(\begin{array}{c}
a \\
a^{\varepsilon} \\
a^{\varepsilon \varepsilon}
\end{array}\right)=-\int d \varepsilon v(\varepsilon) f_{\mathrm{F}}^{\prime}(\varepsilon)\left(\begin{array}{c}
1 \\
\left(\varepsilon-\mu_{0}\right) / T_{0} \\
\left(\varepsilon-\mu_{0}\right)^{2} / T_{0}
\end{array}\right) .
$$

В нашей задаче электрическое поле $\mathbf{E}(\mathbf{r})$ является внешним, поэтому $\operatorname{div} \mathbf{E}=0$. С учетом этого факта и формул (23), (26) и (29) подстановка выражений (24) и (25) в уравнения (27) и (28) приводит к уравнениям баланса:

$\left\{\begin{array}{c}a \frac{\partial \delta \mu}{\partial t}+a^{\varepsilon} \frac{\partial \delta T}{\partial t}+\sigma_{x x}^{\alpha} \Delta \delta \mu+\alpha_{x x}^{\alpha} \Delta \delta T=R \delta \mu+R^{\varepsilon} \delta T \\ T_{0} a^{\varepsilon} \frac{\partial \delta \mu}{\partial t}+a^{\varepsilon \varepsilon} \frac{\partial \delta T}{\partial t}+T_{0} \alpha_{x x}^{\alpha} \Delta \delta \mu+\kappa_{x x}^{\alpha} \Delta \delta T \\ =T_{0} R^{\varepsilon} \delta \mu+\left(R^{\varepsilon \varepsilon}+L\right) \delta T .\end{array}\right.$

Здесь $\Delta=\partial^{2} / \partial x^{2}+\partial^{2} / \partial y^{2}-$ двумерный оператор Лапласа.

Полученную систему уравнений (30) на величины $\delta \mu(\mathbf{r})$ и $\delta T(\mathbf{r})$ нужно дополнить граничными условиями на краях изучаемой двумерной области. Если некоторый участок границы $\partial G^{c}$ является закрытым (частицы не могут пройти через него), то на этом участке потоки в перпендикулярном к нему направлении отсутствуют:

$$
\left.\mathbf{j}^{\alpha}(\mathbf{r}) \cdot \mathbf{n}(\mathbf{r})\right|_{\partial G^{c}}=0,\left.\mathbf{q}^{\alpha}(\mathbf{r}) \cdot \mathbf{n}(\mathbf{r})\right|_{\partial G^{c}}=0 .
$$

Здесь $\mathbf{n}(\mathbf{r})$ - единичная нормаль к кривой $\partial G^{c}$. При этом в соответствии с условием (3) необходимо потребовать, чтобы электрическое поле и градиенты $\nabla \delta \mu$ и $\nabla \delta T$ на этом участке границы удовлетворяли соотношениям $\left.\mathbf{E}(\mathbf{r})\left\|\left.\mathbf{t}(\mathbf{r})\right|_{\partial G^{c}}, \nabla \delta \mu(\mathbf{r})\right\| \mathbf{n}(\mathbf{r})\right|_{\partial G^{c}}, \nabla \delta T(\mathbf{r}) \|\left.\mathbf{n}(\mathbf{r})\right|_{\partial G^{c}}$, где $\mathbf{t}(\mathbf{r})$ - единичная касательная к кривой $\partial G^{c}$. На открытом участке границы $\partial G^{o}$ отсутствуют градиенты всех величин в перпендикулярном к границе направлении: $\left.\nabla \delta \mu(\mathbf{r})\left\|\left.\mathbf{t}(\mathbf{r})\right|_{\partial G^{o}}, \nabla \delta T(\mathbf{r})\right\| \mathbf{t}(\mathbf{r})\right|_{\partial G^{o}}=0$. При этом на поле $\mathbf{E}(\mathbf{r})$ в этой области границы необходимо наложить условие $\mathbf{E}(\mathbf{r}) \|\left.\mathbf{n}(\mathbf{r})\right|_{\partial G^{o}}$.

Из определений (26) видно, что для каждого типа носителей $\alpha=e, h$ диагональные и недиагональные кинетические коэффициенты связаны соотношениями:

$$
\begin{array}{ll}
\sigma_{y y}^{\alpha}=\sigma_{x x}^{\alpha}, & \sigma_{y x}^{\alpha}=-\sigma_{x y}^{\alpha}, \\
\alpha_{y y}^{\alpha}=\alpha_{x x}^{\alpha}, & \alpha_{y x}^{\alpha}=-\alpha_{x y}^{\alpha}, \\
\kappa_{y y}^{\alpha}=\kappa_{x x}^{\alpha}, & \kappa_{y x}^{\alpha}=-\kappa_{x y}^{\alpha} .
\end{array}
$$

При этом диагональные кинетические коэффициенты одинаковы для электронов и дырок, а недиагональные различаются знаком:

$$
\begin{aligned}
& \sigma_{x x}^{e}=\sigma_{x x}^{h}, \quad \sigma_{x y}^{e}=-\sigma_{x y}^{h}, \\
& \alpha_{x x}^{e}=\alpha_{x x}^{h}, \quad \alpha_{x y}^{e}=-\alpha_{x y}^{h}, \\
& \kappa_{x x}^{e}=\kappa_{x x}^{h}, \quad \kappa_{x y}^{e}=-\kappa_{x y}^{h} .
\end{aligned}
$$

Поэтому удобно ввести следующие обозначения кинетических коэффициентов без индекса $\alpha$ :

$$
\begin{aligned}
& \sigma_{x x}=\sigma_{x x}^{\alpha}, \quad \sigma_{x y}=\sigma_{x y}^{h}>0, \\
& \alpha_{x x}=\alpha_{x x}^{\alpha}, \quad \alpha_{x y}=\alpha_{x y}^{h}>0, \\
& \kappa_{x x}=\kappa_{x x}^{\alpha}, \quad \kappa_{x y}=\kappa_{x y}^{h}>0 .
\end{aligned}
$$

С учетом введенных определений (29) и (32) система уравнений (30) приобретет вид

$$
\left\{\begin{array}{l}
a \frac{\partial \delta \mu}{\partial t}+a^{\varepsilon} \frac{\partial \delta T}{\partial t}+\sigma_{x x} \Delta \delta \mu+\alpha_{x x} \Delta \delta T=R \delta \mu+R^{\varepsilon} \delta T \\
a^{\varepsilon} \frac{\partial \delta \mu}{\partial t}+\widetilde{a}^{\varepsilon \varepsilon} \frac{\partial \delta T}{\partial t}+\alpha_{x x} \Delta \delta \mu+\widetilde{\kappa}_{x x} \Delta \delta T=R^{\varepsilon} \delta \mu+\tilde{L} \delta T .
\end{array}\right.
$$

Здесь введены обозначения: $\widetilde{a}^{\varepsilon \varepsilon}=a^{\varepsilon \varepsilon} / T_{0}, \widetilde{\kappa}_{x x}=\kappa_{x x} / T_{0}$, $\widetilde{L}=\left(R_{T}^{\varepsilon}+L\right) / T_{0}$. Граничные условия на замкнутой границе (31) можно переписать в виде

$$
\left\{\begin{array}{l}
{\left.\left[\sigma_{x y} e \mathbf{E} \cdot \mathbf{t}-\left(\sigma_{x x} \nabla \delta \mu+\alpha_{x x} \nabla \delta T\right) \cdot \mathbf{n}\right]\right|_{\partial G^{c}}=0,} \\
{\left.\left[\alpha_{x y} e \mathbf{E} \cdot \mathbf{t}-\left(\alpha_{x x} \nabla \delta \mu+\widetilde{\kappa}_{x x} \nabla \delta T\right) \cdot \mathbf{n}\right]\right|_{\partial G^{c}}=0 .}
\end{array}\right.
$$

Тот факт, что балансовые уравнения (33) и граничные условия (34) для каждого их типов носителей получились одинаковыми, означает, что решение, сохраняющее зарядовую нейтральность (соотношения $\delta \mu^{e}=\delta \mu^{h}$, $\left.\delta T^{e}=\delta T^{h}\right)$, существует. Тем самым мы показали, что выполнение условия (3) на границе области обеспечивает существование решения, сохраняющего это условие и зарядовую нейтральность всюду внутри образца. 


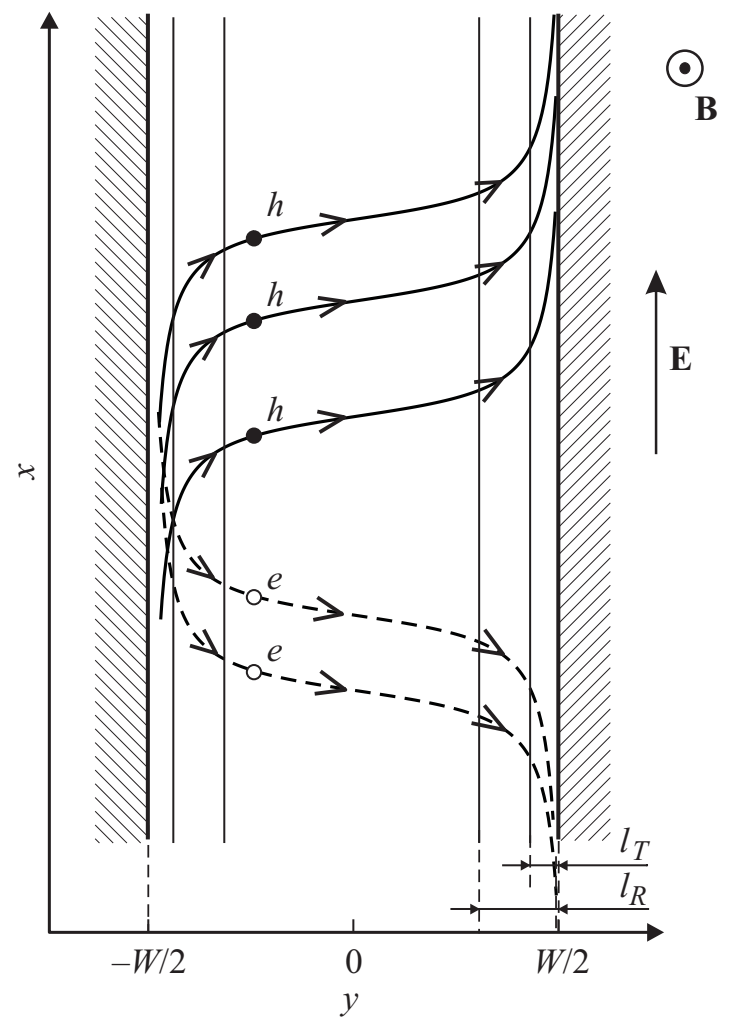

Рис. 1. Образец, имеющий форму длинной полосы; направления электрического и магнитного полей, а также направления потоков электронов $(e)$ и дырок $(h)$.

\section{4. Решение уравнений баланса}

Решим уравнения (33) с граничными условиями (34) для случая области, имеющей форму полосы длиной $L$ и шириной $W \ll L$ (см. рис. 1$)$. Будем считать боковые края полосы, $y= \pm W / 2$, непроницаемыми для частиц, а удаленные края, $x \rightarrow \pm \infty$, - открытыми. Внешнее электрическое поле $\mathbf{E}$ будем считать однородным и направленным вдоль канала. Рассмотрим стационарный режим. Все величины зависят только от координаты $y$, поэтому уравнения баланса являются одномерными и могут быть решены аналитически. Ясно, что при этом условие (3) выполняется тривиальным образом.

Важную роль играют характерная длина, на которой релаксирует возмущение температуры, $l_{T}=\sqrt{\sigma_{x x} / \widetilde{L}}$ (тепловая длина), и характерная длина, на которой релаксирует возмущение концентрации, $l_{R}=\sqrt{\sigma_{x x} / R}$ (рекомбинационная длина). Введем также обозначения $l_{T}^{0}$ и $l_{R}^{0}$ для тепловой и рекомбинационной длин в нулевом магнитном поле.

В настоящей статье приведем только окончательные результаты для частного, но важного случая, когда рекомбинация происходит много медленнее охлаждения, $\widetilde{L} \gg R, R^{\varepsilon}$, что соответствует неравенству $l_{T} \ll l_{R}$. Мы получим в этом режиме формулы для распределений химического потенциала, температуры и плотности электрического тока, а также магнетосопротивления.
Детальный анализ поведения магнетосопротивления в настоящей статье мы проведем только для случая узкого образца, $W \ll l_{T}^{0}$.

Соотношения (24) и (25) для потоков частиц и тепла приобретают вид

$$
\begin{gathered}
j_{x}^{\alpha}=\sigma_{x x} q^{\alpha} E-\sigma_{x y}^{\alpha} \delta \mu^{\prime}-\alpha_{x y}^{\alpha} \delta T^{\prime}, \\
j_{y}^{\alpha}=-\sigma_{x y} e E-\sigma_{x x} \delta \mu^{\prime}-\alpha_{x x} \delta T^{\prime}, \\
q_{y}^{\alpha}=-T_{0} \alpha_{x y} e E-T_{0} \alpha_{x x} \delta \mu^{\prime}-\kappa_{x x} \delta T^{\prime} .
\end{gathered}
$$

Уравнения баланса (33) становятся обыкновенными дифференциальными уравнениями

$$
\left\{\begin{array}{l}
\sigma_{x x} \delta \mu^{\prime \prime}+\alpha_{x x} \delta T^{\prime \prime}=R \delta \mu+R_{T} \delta T, \\
\alpha_{x x} \delta \mu^{\prime \prime}+\widetilde{\kappa}_{x x} \delta T^{\prime \prime}=R^{\varepsilon} \delta \mu+\widetilde{L} \delta T,
\end{array}\right.
$$

а условия (34) на границах $y= \pm W / 2$ имеют вид

$$
\left\{\begin{array}{l}
{\left.\left[\sigma_{x x} \delta \mu^{\prime}+\alpha_{x x} \delta T^{\prime}\right]\right|_{y= \pm W / 2}=-\sigma_{x y} e E} \\
{\left.\left[\alpha_{x x} \delta \mu^{\prime}+\widetilde{\kappa}_{x x} \delta T^{\prime}\right]\right|_{y= \pm W / 2}=-\alpha_{x y} e E .}
\end{array}\right.
$$

Из уравнений (35) видно, что плотность электрического тока $\mathbf{J}=q^{e} \mathbf{j}^{e}+q^{h} \mathbf{j}^{h}$ направлена вдоль канала, $\mathbf{J}=(J, 0)$, и определяется выражением

$$
J(y)=2\left[e^{2} \sigma_{x x} E-e \sigma_{x y} \delta \mu^{\prime}(y)-e \alpha_{x y} \delta T^{\prime}(y)\right] .
$$

Экспериментально измеряемой величиной является усредненное по ширине канала значение плотности тока

$$
\bar{J}=\frac{1}{W} \int_{-W / 2}^{W / 2} d y J(y) .
$$

Сопротивление канала определяется как $R_{\square}=E / \bar{J}$.

Решение уравнений баланса было нами выполнено по методу, разработанному в работе [23], с использованием аппарата линейной алгебры (вычисление аналитических функций от матриц).

Распределения химического потенциала, температуры и плотности электрического тока в режиме медленной рекомбинации, $R, R^{\varepsilon} \ll \widetilde{L}$, имеют вид

$$
\begin{aligned}
\delta \mu(y)= & -e E\left[l_{R} \beta^{0} \frac{\sinh \left(y / l_{R}\right)}{\cosh \left(W / 2 l_{R}\right)}\right. \\
& \left.+l_{T} c^{\varepsilon} \frac{c^{\varepsilon} \beta^{0}-\beta^{\varepsilon}}{c^{\varepsilon \varepsilon}-\left(c^{\varepsilon}\right)^{2}} \frac{\sinh \left(c^{\prime} y / l_{T}\right)}{c^{\prime} \cosh \left(c^{\prime} W / 2 l_{T}\right)}\right], \\
\delta T(y)= & e E l_{T} \frac{c^{\varepsilon} \beta^{0}-\beta^{\varepsilon}}{c^{\varepsilon \varepsilon}-\left(c^{\varepsilon}\right)^{2}} \frac{\sinh \left(c^{\prime} y / l_{T}\right)}{c^{\prime} \cosh \left(c^{\prime} W / 2 l_{T}\right)}, \\
J(y)= & 2 e^{2} \sigma_{x x} E\left[1+\left(\beta^{0}\right)^{2} \frac{\cosh \left(y / l_{R}\right)}{\cosh \left(W / 2 l_{R}\right)}\right. \\
& \left.+\frac{\left(c^{\varepsilon} \beta^{0}-\beta^{\varepsilon}\right)^{2}}{c^{\varepsilon \varepsilon}-\left(c^{\varepsilon}\right)^{2}} \frac{\cosh \left(c^{\prime} y / l_{T}\right)}{c^{\prime} \cosh \left(c^{\prime} W / 2 l_{T}\right)}\right],
\end{aligned}
$$

где $\quad c^{\varepsilon}=\alpha_{x x} / \sigma_{x x}, \quad c^{\varepsilon \varepsilon}=\widetilde{\kappa}_{x x} / \sigma_{x x}, \quad \beta^{0}=\sigma_{x y} / \sigma_{x x}$, $\beta^{\varepsilon}=\alpha_{x y} / \sigma_{x x}, \quad c^{\prime}=\left[c^{\varepsilon \varepsilon}-\left(c^{\varepsilon}\right)^{2}\right]^{-1 / 2}$. Заметим, что в 

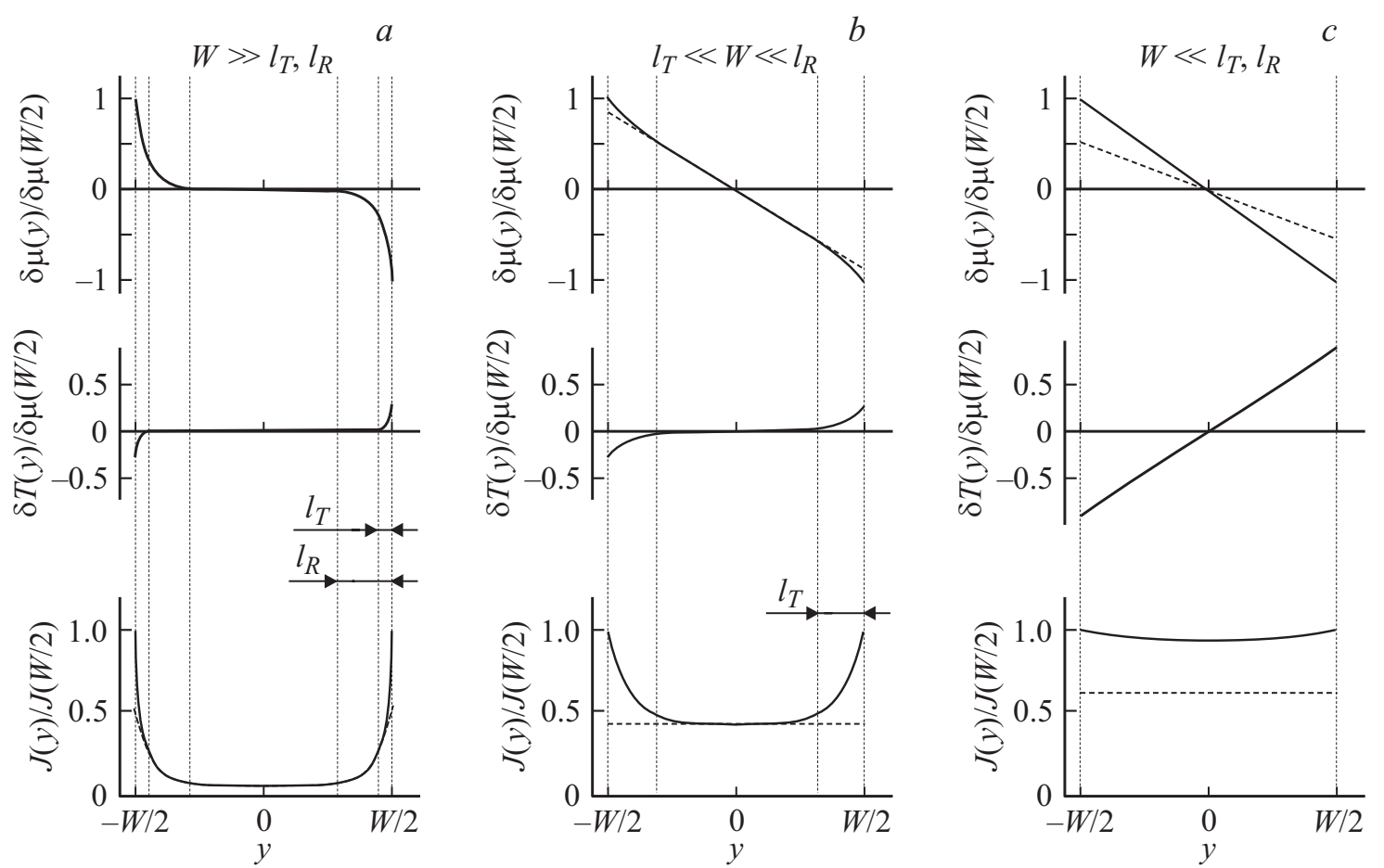

Рис. 2. Распределения химического потенциала, температуры и плотности электрического тока в широком $(a)$, среднем $(b)$ и узком $(c)$ образцах (сплошные кривые). Штриховые кривые - результаты расчета химического потенциала и плотности электрического тока без учета возмущения температуры (в соответствии с рассмотрением работы [22]).

рассматриваемом приближении величиа $R^{\varepsilon}$ не вошла в результаты (38)-(40). С помощью формул (26) можно показать, что величина $c^{\varepsilon \varepsilon}-\left(c^{\varepsilon}\right)^{2}$ всегда положительна, а величина $c^{\varepsilon} \beta^{0}-\beta^{\varepsilon}$ положительна для случая $d \beta / d \varepsilon<0$ (здесь $\beta(\varepsilon)=\omega_{c}(\varepsilon) \tau(\varepsilon)$ ) и отрицательна при $d \beta / d \varepsilon>0$. Мы будем считать, что $d \beta / d \varepsilon<0$ и оба слагаемых в формуле (38) имеют одинаковый знак.

Опишем поведение распределений (38)-(40). Рассмотрим случай, типичный для невырожденного полупроводника, когда величины $c^{\varepsilon}, c^{\varepsilon \varepsilon}, c^{\prime}$ порядка единицы, но не равны друг другу и, аналогично, $\beta^{0} \sim \beta^{\varepsilon}$, но $\beta^{0} \neq \beta^{\varepsilon}$. Такая ситуация реализуется, например, для однослойного и двухслойного графена в точке электронейтральности при достаточно высокой температуре. Обозначим через $\beta$ характерное значение параметра $\beta(\varepsilon)$ при $\varepsilon \sim \max \left(\mu_{0}, T_{0}\right)$. Таким способом введенный параметр $\beta \sim B$ характеризует силу магнитного поля $B$; он есть величина того же порядка, что $\beta^{0}$ и $\beta^{\varepsilon}$. Необходимо различать случаи широкого образца, $W \gg l_{R}$, образца средней ширины, $l_{T} \ll W \ll l_{R}$, и узкого образца, $W \ll l_{T}$.

Для широкого образца обе функции $\delta \mu(y)$ и $\delta T(y)$ в центральной части образца экспоненциально малы. В окрестности краев в слое шириной $l_{T}$ температура $\delta T(y)$ возрастает до значений $\pm e E l_{T} \beta$. Распределение $\delta \mu(y)$ вблизи краев в слое шириной $l_{R}$ возрастает до значения $\pm e E l_{R} \beta$.

Для образца средней ширины распределение температуры такое же, как и для широкого образца. Распре- деление $\delta \mu(y)$ внутри полосы есть линейная функция, $e E \beta y$. В узкой области вблизи краев шириной порядка $l_{T}$ функция $\delta \mu(y)$ слегка возрастает по модулю на величину $e E \beta l_{T} \ll e E \beta W / 2$.

Для узкого образца температура и химический потенциал линейно зависят от координаты $y$, причем направления их изменения противоположны: $\delta \mu(y) \propto-e E \beta y$, $\delta T(y) \propto e E \beta y$.

Таким образом, только для узкого образца возмущения $\delta \mu$ и $\delta T$ имеют один порядок величины, в остальных случаях $\delta \mu \gg \delta T$. Для широкого и среднего образцов, $W \gg l_{T}$, возмущения температуры существенны только вблизи краев в областях шириной $\sim l_{T}$.

В силу уравнения (40) распределение плотности электрического тока для узкого образца близко к однородному:

$J(y)=2 e^{2} \sigma_{x x} E\left[1+\left(\beta^{0}\right)^{2}+\frac{\left(c^{\varepsilon} \beta^{0}-\beta^{\varepsilon}\right)^{2}}{c^{\varepsilon \varepsilon}-\left(c^{\varepsilon}\right)^{2}}\left(1-\frac{c^{\prime 2}}{l_{T}^{2}} y^{2}\right)\right]$.

Малая поправка, пропорциональная $y^{2}$, обусловлена термоэлектрическим эффектом. Для образца средней толщины распределение плотности тока внутри образца преимущественно однородно и имеет величину $2 e^{2} \sigma_{x x} E\left(1+\beta^{2}\right)$. Вблизи краев образца в слое толщиной $\sim l_{T}$ плотность тока возрастает на величину $\sim 2 e^{2} \sigma_{x x} E \beta^{2}$. Для широкого образца ток внутри образца имеет значение $2 e^{2} \sigma_{x x} E$, что для классически сильного магнитного поля, $\beta \gg 1$, значительно меньшее его 
значений в приграничных областях, которые по порядку величины равны $2 e^{2} \sigma_{x x} E \beta^{2}$.

Описанное поведение $\delta \mu(y), \delta T(y)$ и $J(y)$ показано на рис. 2.

Для усредненного тока в размерных обозначениях получаем:

$$
\begin{aligned}
\bar{J}= & 2 e^{2} E \sigma_{x x}\left[1+\frac{\tanh \left(W / 2 l_{R}\right)}{W / 2 l_{R}} \frac{\sigma_{x y}^{2}}{\sigma_{x x}^{2}}\right. \\
& \left.+\frac{\tanh \left(c^{\prime} W / 2 l_{T}\right)}{c^{\prime} W / 2 l_{T}} \frac{\left[\sigma_{x y} \alpha_{x x}-\alpha_{x y} \sigma_{x x}\right]^{2}}{\sigma_{x x}^{2}\left[\widetilde{\kappa}_{x x} \sigma_{x x}-\left(\alpha_{x x}\right)^{2}\right]}\right],
\end{aligned}
$$

где $c^{\prime}=\sigma_{x x} / \sqrt{\widetilde{\kappa}_{x x} \sigma_{x x}-\left(\alpha_{x x}\right)^{2}}$. Проанализируем зависимость $\bar{J}$ от магнитного поля $B$ для образца, являющегося узким при $B=0$, т.е. ширина которого удовлетворяет неравенству $W \ll l_{T}^{0}$.

В режиме малого магнитного поля, когда для всех частиц $\beta(\varepsilon)=\omega_{c}(\varepsilon) \tau(\varepsilon) \ll 1$, формула $(41)$ приводит к малой отрицательной поправке к току, квадратичной по магнитному полю:

$$
\bar{J}=J_{0}\left[1-\frac{\left\langle\Delta x^{2}\right\rangle\left\langle\Delta \beta^{2}\right\rangle-\langle\Delta x \Delta \beta\rangle^{2}}{\left\langle\Delta x^{2}\right\rangle}\right],
$$

где $J_{0}=2 e^{2} E \sigma_{0}-$ ток в нулевом магнитном поле, $\sigma_{0}=\int d \varepsilon v(\varepsilon)\left[-f_{\mathrm{F}}^{\prime}(\varepsilon)\right]\left[v(\varepsilon)^{2} / 2\right] \tau(\varepsilon), \Delta x=x(\varepsilon)-\langle x\rangle$, $\Delta \beta=\beta(\varepsilon)-\langle\beta\rangle, x=\left(\varepsilon-\mu_{0}\right) / T_{0}$, скобки $\langle\ldots\rangle$ означают операцию усреднения:

$$
\langle A(\varepsilon)\rangle=\frac{1}{\sigma_{0}} \int d \varepsilon v(\varepsilon)\left[-f_{\mathrm{F}}^{\prime}(\varepsilon)\right] \frac{v(\varepsilon)^{2}}{2} \tau(\varepsilon) A(\varepsilon) .
$$

В формуле (42) выражение в числителе дроби пропорционально $B^{2}$ и всегда неотрицательно в силу неравенства Коши-Буняковского, поэтому магнетосопротивление за счет термоэлектрического эффекта для обычной двухкомпонентной ферми-системы в малых полях всегда положительно или отсутствует. Нулевое магнетосопротивление реализуется, когда зависимость диагональных кинетических коэффициентов от магнитного поля описывается друдевским фактором $\left[1+\beta\left(\mu_{0}\right)^{2}\right]^{-1}$, а недиагональных - фактором $\beta\left(\mu_{0}\right) /\left[1+\beta\left(\mu_{0}\right)^{2}\right]$. Это имеет место при вырождении электронов и дырок, когда $\mu_{0} \neq 0$ и $T_{0} \ll \mu_{0}$, а также при такой зависимости циклотронной частоты и времени релаксации от энергии, что $\beta(\varepsilon) \equiv$ const. Последнее имеет место, например, для однослойного графена с заряженными примесями.

В промежуточных магнитных полях, в которых при одних энергиях $\beta(\varepsilon) \gg 1$, а при других $\beta(\varepsilon) \ll 1$, зависимость $\bar{J}(B)$ может определяться конкретными особенностями спектра носителей $\varepsilon_{p}$. Для широкого образца однослойного графена, в котором основной вклад в ток происходит от центральной части образца и описывается объемным тензором проводимости $\hat{\sigma}^{\alpha}$, результирующее магнетосопротивление в таком режиме оказывается корневым: $R_{\square}(B)-R_{\square}(0) \propto \sqrt{B}$ [33].

Изучим зависимость $\bar{J}(B)$ в классически сильном магнитном поле, когда $\beta(\varepsilon) \gg 1$ при всех энергиях $\varepsilon$. В этом пределе кинетические коэффициенты и характерные длины имеют следующие асимптотики: $\sigma_{x x} \rightarrow S_{x x} / \beta^{2}$, $\alpha_{x x} \rightarrow A_{x x} / \beta^{2}, \widetilde{\kappa}_{x x} \rightarrow K_{x x} / \beta^{2}, \sigma_{x y} \rightarrow S_{x y} / \beta, \alpha_{x y} \rightarrow A_{x y} / \beta$, $l_{T} \rightarrow L_{T} / \beta, l_{R} \rightarrow L_{R} / \beta$. Здесь величина $\beta=\langle\beta(x)\rangle \propto B$ имеет тот же смысл, что и ранее, и характеризует силу магнитного поля. Ниже мы снова будем обсуждать только случай невырожденной двумерной системы, $\mu_{0} \ll T_{0}$, когда все коэффициенты $S_{x x}, A_{x x}, K_{x x}, S_{x y}$ и $A_{x y}$ одного порядка величины. В этом пределе из формулы (41) получаем:

$$
\bar{J}(\beta)=J_{1}\left[F\left(\frac{\beta}{\beta_{R}}\right)+q F\left(\frac{\beta}{\beta_{T}}\right)\right] .
$$

Здесь $J_{1}=2 e^{2} E S_{x y}^{2} / S_{x x}-$ величина того же порядка, что и $J_{0}$ в формуле (42); $\beta_{T}=2 L_{T} / W c_{\infty}^{\prime} \gg 1$ и $\beta_{R}=2 L_{R} / W \gg \beta_{T}-$ характерные значения параметра $\beta$, при которых происходит смена режимов в поведении $\bar{J}(\beta)$; коэффициенты $c_{\infty}^{\prime}=S_{x x} / \sqrt{K_{x x} S_{x x}-A_{x x}^{2}}$ и $q=\left(A_{x x} S_{x y}-A_{x y} S_{x x}\right)^{2} /\left(K_{x x} S_{x x}-A_{x x}^{2}\right) S_{x y}^{2}$ - величины порядка единицы; функция $F(z)=\tanh (z) / z$ имеет простые асимтотики:

$$
F(z)=\mid \begin{aligned}
& 1-z^{2} / 3, \quad z \rightarrow 0 \\
& 1 / z, \quad z \rightarrow \infty
\end{aligned} .
$$

Из формулы (43) видно, что зависимость тока от магнитного поля различна в областях $1 \ll \beta \ll \beta_{T}$, $\beta_{T} \ll \beta \ll \beta_{R}, \beta \gg \beta_{R}$. В области $1 \ll \beta \ll \beta_{T}$ с учетом неравенства $L_{T} \ll L_{R}$ асимптотика (44) дает

$$
\bar{J}(\beta)=J_{1}\left(1+q-\frac{q}{3} \frac{\beta^{2}}{\beta_{T}^{2}}\right) .
$$

В области $\beta_{T} \ll \beta \ll \beta_{R}$ асимптотика (44) дает

$$
\bar{J}(\beta)=J_{1}\left(1-\frac{1}{3} \frac{\beta^{2}}{\beta_{R}^{2}}+\frac{q \beta_{T}}{\beta}\right) .
$$

Из формулы видно, что в этой области полей существует пограничное значение параметра $\beta, \beta_{T R}=\sqrt[3]{3 q \beta_{T} \beta_{R}^{2}}$. $\mathrm{B}$ магнитных полях $\beta_{T} \ll \beta \ll \beta_{T R}$ зависимость тока от магнитного поля определяется преимущественно термоэлектрическим эффектом (слагаемое $q \beta_{T} / \beta$ ), в то время как в магнитных полях $\beta_{T R} \ll \beta \ll \beta_{R}$ поведение $\bar{J}(\beta)$ контролируется преимущественно процессами рекомбинации (слагаемое $-\beta^{2} / 3 \beta_{R}^{2}$ ). В области $\beta \gg \beta_{R}$ из формул (43) и (44) аналогично получаем:

$$
\bar{J}(\beta)=J_{1} \frac{\beta_{R}}{\beta} .
$$

Описанная зависимость среднего тока $\bar{J}(\beta)$ от магнитного поля $\beta$ приводит к зависимости сопротивления, $R_{\square}(\beta)=E / \bar{J}(\beta)$, от $\beta$ приведенной на рис. 3 .

Поведение магнетосопротивления в области полей $\beta \gg \beta_{T R}$ с точностью до величин порядка $L_{T} / L_{R}$ оказывается таким же, как было получено в работе [22] без учета возмущения температуры: очень медленный 

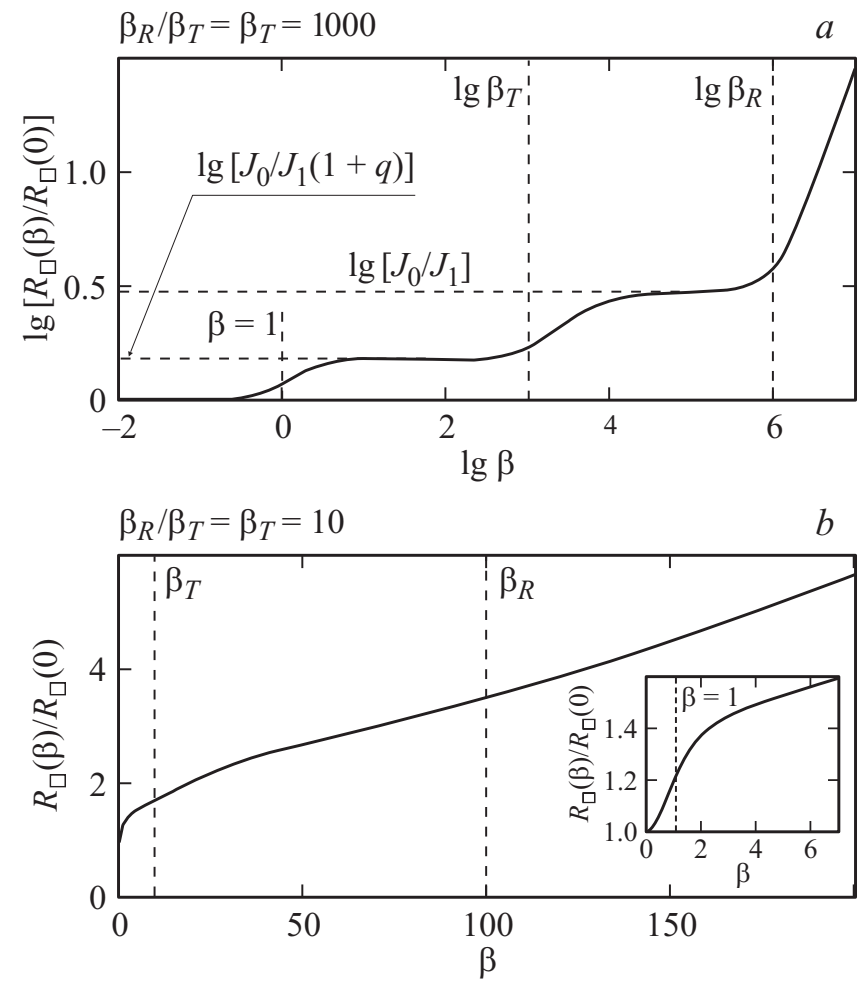

Рис. 3. Зависимости относительной величины сопротивления от магнитного поля для узкого образца при очень больших $(a)$ и умеренно больших $(b)$ значениях параметров $\beta_{R}$ и $\beta_{T}$.

квадратичный рост $R_{\square}(\beta)$ при $\beta<\beta_{R}$ сменяется линейным ростом, $R_{\square}(\beta) \propto \beta$, при $\beta>\beta_{R}$ (см. рис. 3). В области полей $\beta \ll \beta_{T R}$ зависимость $R_{\square}(\beta)$ имеет нетривиальный характер. При классически слабых и умеренных магнитных полях, $\beta \sim 1$, сопротивление $R_{\square}$ возрастает на величину порядка своего значения при $\beta=0$ и насыщается при $\beta \gg 1$. При переходе параметром $\beta$ значения $\beta_{T}$ сопротивление $R_{\square}$ еще возрастает на величину порядка $R_{\square}(0)$ и затем снова насыщается при $\beta \gg \beta_{T}$.

Однако такое ступенеобразное поведение магнетосопротивления $R_{\square}(B)$ реализуется только в случае, когда величины $W, L_{T}$ и $L_{R}$ имеют очень сильно различающиеся порядки величины (см. рис. $3, a)$. Если порядки величин $W, L_{T}$ и $L_{R}$ относительно близки (хотя и выполнено неравенство $\left.W \ll L_{T} \ll L_{R}\right)$, поведение функции $R_{\square}(B)$ является более плавным: без участков насыщения, но с изменением характера кривизны $R_{\square}(B)$ на различных участках (см. рис. $3, b)$.

\section{5. Заключение}

В настоящей работе изучен магнетотранспорт в двухкомпонентной системе в точке электронейтральности при наличии неоднородных по образцу распределений потоков частиц, их температур и концентраций.

В случае, если рекомбинация электронов и дырок происходит значительно медленнее их теплообмена с фононами, в достаточно широком образце распределения электронов и дырок и результирующее магнетосопротивление определяются диффузией и рекомбинацией.

В достаточно узком образце реализуется режим, в котором определяющую роль играют процессы теплообмена и теплопереноса. Существенный вклад в электрический ток дают неравновесные потоки электронов и дырок, связанные с градиентом температуры. Величины таких потоков определяются балансом между термоэлектрическими эффектами, теплопроводностью и теплообменом с равновесными фононами. Результирующее сопротивление как функция магнитного поля растет с изменяющейся скоростью, имеет несколько участков насыщения и в достаточно больших магнитных полях выходит на режим линейного роста.

Работа выполнена при поддержке грантов президента Российской Федерации МК-8826.2016.2, грантов РФФИ 16-02-01166-а и 17-02-00217-а и гранта EU Network FP7-PEOPLE-2013-IRSES, Grant N 612624 „InterNoM“.

\section{Список литературы}

[1] A.T. Hatke, M.A. Zudov, J.L. Reno, L.N. Pfeiffer, K.W. West. Phys. Rev. B, 85, 081304 (2012).

[2] R.G. Mani, A. Kriisa, W. Wegscheider. Sci. Rep., 3, 2747 (2013).

[3] L. Bockhorn, P. Barthold, D. Schuh, W. Wegscheider, R.J. Haug. Phys. Rev. B, 83, 113301 (2011).

[4] Q. Shi, P.D. Martin, Q.A. Ebner, M.A. Zudov, L.N. Pfeiffer, K.W. West. Phys. Rev. B, 89, 201301 (2014).

[5] I.A. Dmitriev, A.D. Mirlin, D.G. Polyakov, M.A. Zudov. Rev. Mod. Phys., 84, 1709 (2012).

[6] A.L. Friedman, J.L. Tedesco, P.M. Campbell, J.C. Culbertson, E. Aifer, F.K. Perkins, R.L. Myers-Ward, J.K. Hite, C.R. Eddy, G.G. Jernigan, D.K. Gaskill. Nano Lett., 10, 3962 (2010).

[7] R.S. Singh, X.Wang, W.C. Ariando, A.T.S. Wee. Appl. Phys. Lett., 101, 183105 (2012).

[8] M. Veldhorst, M. Snelder, M. Hoek, C.G. Molenaar, D.P. Leusink, A.A. Golubov, H. Hilgenkamp, A. Brinkman. Phys. Status Solidi RRL, 7, 26 (2013).

[9] W. Wang, Y. Du, G. Xu, X. Zhang, E. Liu, Z. Liu, Y. Shi, J. Chen, G. Wu, X. Zhang. Sci. Rep., 3, 2181 (2013).

[10] G.M. Gusev, E.B. Olshanetsky, Z.D. Kvon, N.N. Mikhailov, S.A. Dvoretsky. Phys. Rev. B, 87, 081311 (2013).

[11] F. Kisslinger, C. Ott, C. Heide, E. Kampert, B. Butz, E. Spiecker, S. Shallcross, H.B. Weber. Nature Phys., 11, 650 (2015).

[12] S. Wiedmann, A. Jost, C. Thienel, C. Brune, P. Leubner, H. Buhmann, L.W. Molenkamp, J.C. Maan, U. Zeitler. Phys. Rev. B, 91, 205311 (2015).

[13] C.M.Wang, X.L. Lei. Phys. Rev. B, 92, 125303 (2015).

[14] G.Yu. Vasileva, D. Smirnov, Yu.L. Ivanov, Yu.B. Vasilyev, P.S. Alekseev, A.P. Dmitriev, I.V. Gornyi, V.Yu. Kachorovskii, M. Titov, B.N. Narozhny, R.J. Haug. Phys. Rev. B, 93, 195430 (2016).

[15] Квантовый эфбект Холла, под ред. Р.Е. Прейнджа и С.М. Гирвина (М., Мир, 1989).

[16] Y.M. Galperin. Quantum Transport, Lecture Notes (Lund University, 1998). 
[17] Y.V. Nazarov, Y.M. Blanter. Quantum Transport, Introduction to Nanoscience (Cambridge University Press, N. Y., 2009).

[18] A.D. Mirlin, D.G. Polyakov, F. Evers, P. Wolfle. Phys. Rev. Lett., 87, 126805 (2001).

[19] A. Dmitriev, M. Dyakonov, R. Jullien. Phys. Rev. B, 64, 233321 (2001).

[20] V.V. Cheianov, A.P. Dmitriev, V.Y. Kachorovskii. Phys. Rev. B, 68, 201304 (2003).

[21] Y.M. Beltukov, M.I. Dyakonov. Phys. Rev. Lett., 116, 176801 (2016).

[22] P.S. Alekseev, A.P. Dmitriev, I.V. Gornyi, V.Y. Kachorovskii, B.N. Narozhny, M. Schütt, M. Titov. Phys. Rev. Lett., 114, 156601 (2015).

[23] B.N. Narozhny, I.V. Gornyi, M. Titov, M. Schütt, A.D. Mirlin. Phys. Rev. B, 91, 035414 (2015).

[24] M. Hruska, B. Spivak. Phys. Rev. B, 65, 033315 (2002).

[25] P.S. Alekseev. Phys. Rev. Lett., 117, 166601 (2016).

[26] M.V. Cheremisin. ЖЭТФ, 119, 409 (2001).

[27] M.V. Cheremisin. Physica E, 27, 151 (2005).

[28] M.V. Cheremisin. ЖЭТФ, 127, 674 (2005).

[29] M.V. Cheremisin. Physica E, 27, 151 (2005).

[30] В. Карпус. ФТП, 20, 12 (1986).

[31] P.S. Alekseev, M.S. Kipa, V.I. Perel, I.N. Yassievich. JETP, 106, 806 (2008).

[32] M.S. Keepa, P.S. Alekseev, I.N. Yassievich. Semiconductors, 44, 198 (2010).

[33] P.S. Alekseev, A.P. Dmitriev, I.V. Gornyi, V.Y. Kachorovskii. Phys. Rev. B, 87, 65432 (2013); Г.Ю. Васильева, П.С. Алексеев, Ю.Л. Иванов, Ю.Б. Васильев, Д. Смирнов, Х. Шмидт, Р.Ж. Хауг, Ф. Гойдер, Г. Начтвей. Письма ЖЭТФ 96, 519 (2012).

[34] M.M. Müller, S. Sachdev. Phys. Rev. B 78, 115419 (2008); M.S. Foster, I.L. Aleiner. Phys. Rev. B 79, 085415 (2009); D. Svintsov, V. Vyurkov, S. Yurcenko, T. Otsuji, V. Ryzhii. J. Appl. Phys. 111, 083715 (2012).

\section{Classical magnetoresistance of a two-component system induced by the thermoelectric effects}

\author{
P.S. Alekseev ${ }^{1}$, I.V. Gornyi ${ }^{1,2,3}$, A.P. Dmitriev ${ }^{1}$, \\ V.Yu. Kachorovskii ${ }^{1}$, M.A. Semina ${ }^{1}$ \\ ${ }^{1}$ loffe Institute, \\ 194021 St. Petersburg, Russia \\ 2 Institut für Nanotechnologie, \\ Karlsruhe Institute of Technology, \\ 76021 Karlsruhe, Germany \\ ${ }^{3}$ Institut für Theorie der Kondensierten Materie, \\ Karlsruhe Institute of Technology, \\ 76128 Karlsruhe, Germany
}

Abstract We have studied magnetotransport in a two-dimensional two-component system formed by electrons and holes with the same densities. From the classical kinetic equation we derived the balance equations which describe the transfer of carriers and heat in the sample. By solving the balance equation for a long sample, we calculated the distributions of the carrier densities and temperatures as well as the electric current density. At enough high magnetic fields, the carrier temperatures, densities and flows in vicinities of the sample edges becomes different from the ones in the bulk of the sample. This leads to a nontrivial positive magnetoresistance. 